\title{
Krankheit und religiöses Tabu - die Lepra in der mittelalterlich-frühneuzeitlichen Gesellschaft Europas
}

\author{
Von Hermann Hörger
}

Angesichts der 15 Millionen registrierten Leprakranken in der gesamten Welt, von denen etwa 800000 in Leprosorien stationiert leben, und bei einer immerhin mit 30 Millionen veranschlagten Dunkelziffer an Lepraerkrankungen hat Luise Rinser in einem Fernsehbeitrag zugunsten des Aussätzigen-Hilfswerkes die grundlegenden Probleme der Lepra heute aufgegriffen. Ihre Ausführungen trugen den Titel «Dem Tode geweiht?». ${ }^{1}$

Neben der körperlichen Not der Kranken und der Pflicht, ihnen zu helfen, sprach Frau Rinser von den weitaus schwerer wiegenden seelischen Gefährdungen, denen die Kranken ausgesetzt sind und die von einer rein finanziellen Leprahilfe nie erreicht und eingegrenzt werden können. Sie forderte neben dem Abbau von Vorurteilen ${ }^{2}$ die Solidarisierung mit den Kranken als Voraussetzung für eine wirksamere Bekämpfung dieser Krankheit von der Basis des Mitmenschlichen her.

Frau Rinser hat damit zweifellos eine sehr wunde Stelle berührt, die über den Einzelmenschen hinaus die gesamte Gesellschaft angeht: eine Gesellschaft, der mit Hilfe der Sozialwissenschaften eine Reihe von Lebensfragen verstehbarer geworden ist, der andererseits aber der Vorwurf nicht erspart bleibt, insbesondere der Krankheit und dem Tode gegenüber eine zunehmend indifferente Haltung einzunehmen, ja beide Bereiche allmählich aus ihrem Bewußtsein zu verdrängen ${ }^{3}$.

Es stimmt, daß schwere Krankheiten und ihre Behandlung weitgehend Institutionen anvertraut worden sind, die zwar von der Gesellschaft getragen werden, dem einzelnen aber den Anblick des Sterbens oder die lange währende Begegnung mit dem unheilbar Kranken abnehmen. Krankheit, vor allem die nicht mehr heilbare, und das Ertragen von Krankheit werden als Gemeinschaftsaufgabe nicht mehr erlebt. Angesichts dieser Tatsache wird die Forderung nach Solidarisierung mit dem Kranken, in unserem Falle mit dem Leprakranken, zu einem ernsten Problem. Es erhebt sich die Frage, wie Menschen vergangener Jahrhunderte der Lepra gegenüberstanden, die sie mehr als jede andere Krankheit als von Gott gesandt glaubten. Soviel 
kann hier bereits vorweggenommen werden, daß es selbst zu Zeiten epidemischer Seuchen zu keinem zwangsweisen Ausschluß des Kranken aus der christlichen Gesellschaft gekommen ist und man sich aus gläubiger Verantwortung heraus dem Kranken noch sehr wohl verpflichtet wußte. Einzig bei der Lepra tritt die zwangsweise Ausgemeinschaftung des Kranken in Erscheinung, und dies bereits in vorchristlicher Zeit ${ }^{4}$. Bis ins 16. Jahrhundert hinein war diese Krankheit, theoretisch zumindest ${ }^{5}$, immer wieder Gegenstand religiöser Tabuisierungen, mit deren Hilfe man sich vor dem Kranken und vor der Krankheit selbst zu schützen hoffte ${ }^{6}$.

Die von Aretaios geschilderte griechisch-römische Übung, an Elephantiasis Erkrankte auszustoßen, scheint in den Jahrhunderten der Spätantike bis zu Beginn des Frühmittelalters nur bedingt übernommen worden zu sein. Denn Jahrhunderte vor den Kreuzzügen ${ }^{7}$ tauchen insbesondere in französischen Synodalerlassen Lepraverordnungen auf, die sich nur auf die Sorge um die Erkrankten beziehen und nicht deren Isolierung aus der menschlichen Gemeinschaft zum Gegenstand haben ${ }^{8}$. Allerdings schränkte die Synode von Lyon (583) bereits die Freizügigkeit der Erkrankten ein. Das Capitulare III des Jahres 789 ordnet aber dann bereits die Absonderung der Leprösen an ${ }^{9}$.

Allerdings lassen die zahlreichen Heilungen Lepröser, die in den Lebensbeschreibungen von Heiligen der Merowingerzeit stereotyp wiederkehren ${ }^{10}$, den Schluß zu, daß diese Krankheit ein schwerwiegendes Problem innerhalb der jeweiligen Gesellschaft darstellte, in welcher der Heilige lebte und wirkte ${ }^{11}$; ohne daß hierfür der Beweis angetreten werden könnte, darf doch die Vermutung geäußert werden, daß die Krankheit und das Krankheitsbewußtsein dieser Zeit schon in greifbare Nähe zum religiösen Tabu getreten waren, welches uns Jahrhunderte später in den liturgischen Erlassen einzelner französischer Bistümer in Gestalt ausführlicher Sequestrationsriten entgegentritt. Zu beachten bleibt freilich für diese wie auch spätere Jahrhunderte, daß die Intensität des Krankheitsbewußtseins nicht ohne weiteres einer adäquaten Verbreitung der Krankheit entsprochen hat ${ }^{12}$.

In der Kreuzzugszeit ${ }^{13 a}$ und den darauffolgenden Jahrhunderten intensiver Handelsbeziehungen mit dem Orient tritt die Krankheit und was aus mangelndem medizinischem Wissen unter dem Krankheitsbegriff «Lepra» seit ältester Zeit subsumiert wurde ${ }^{13 b}$, in erhöhtem Maße auf, so daß eine Verschärfung bestehender Maßnahmen und eine Reihe von neuen Verordnungen zur Eindämmung der Krankheit notwendig werden. Es konnte sich so der landläufige Eindruck verfestigen, die Lepra sei überhaupt erst mit den 
Kreuzzügen in Europa verbreitet worden ${ }^{14}$. Mit der raschen Ausweitung der Krankheit auch über ganz Mittel- und Nordeuropa ${ }^{15}$ ist eine Wende in ihrer Beurteilung im öffentlichen Bewußtsein festzustellen, wie noch näher auszuführen sein wird.

Die frühen fränkischen Synodalerlasse treffen lediglich gewisse Sicherheitsvorkehrungen, um den gesunden Bevölkerungsteil zu schützen, wie etwa die Sicherstellung des Lebensunterhaltes, um bettelndes Umherziehen der Leprakranken zu vermeiden, oder aber auch die generelle Einschränkung von deren Freizügigkeit. An eine strikte Durchführung dieser Erlasse ist indes kaum zu denken, weil diese wiederholt in Erinnerung gerufen werden mußten und für eine strenge Absonderung der Kranken demnach noch kein unmittelbarer Anlaß bestand. Anscheinend genügten die einfachen, prägnanten, aber doch stets im Gedächtnis wachgehaltenen Schutzmaßnahmen, oder aber auch trat die Krankheit noch nicht in einer Häufigkeit auf, daß geschlossenes Vorgehen dagegen notwendig gewesen wäre. Allerdings hatte die Lepra und alle Hautkrankheiten, die dafür gehalten wurden, zu dieser Zeit bereits Eingang in Schwur- und Racheformeln zur Sanktion von Rechtsübertretungen gefunden ${ }^{16}$, was nicht nur auf ihren abschreckenden Charakter, sondern vielleicht auch auf ihre allmählich als Bedrohung empfundene Ausbreitung hin gedeutet werden könnte; das Krankheitsbewußtsein ist hierdurch, unabhängig von der tatsächlichen Verbreitung der Krankheit, mit Sicherheit intensiviert und sensibilisiert worden.

Lediglich das langobardische ${ }^{17}$ und das fränkische Recht ${ }^{18}$ kannten die Möglichkeit der Ehenichtigkeitserklärung im Falle einer Lepraerkrankung, obwohl nirgendwo vermerkt ist, ob und in welchem Umfang von dieser Möglichkeit Gebrauch gemacht worden ist. Reaktionen gegen diesen Rechtsbrauch treten erst relativ spät auf ${ }^{19}$. Sie lassen einmal den Schluß auf eine verstärkte Ausbreitung der Krankheit zu, dann aber auch schon an die Möglichkeit denken, daß durch wachsende diagnostische Sicherheit in der Behandlung der Lepra deren Krankheitsbewußtsein kritisch hinterfragt wird.

Bevor wir uns den kirchlichen Riten der Ausgemeinschaftung der Leprakranken zuwenden, ist kurz auf das öffentliche Bewußtsein in der Beurteilung der Krankheit einzugehen. Es ist zu eruieren, welche Vorstellungsgehalte sich in einer Sprache mit der Krankheit verbinden, die allenthalben als Gottesgeißel empfunden, aber nur in begrenztem Bereich auch so genannt wurde. Die Vollständigkeit der Sprachzeugnisse kann nicht 
gewährleistet werden; aber schon wenige Beispiele verdeutlichen die Vielfalt der aus den unterschiedlichen Erscheinungsformen der Krankheit abgeleiteten Vorstellungsbilder, die in einer Gesellschaft lebendig sind ${ }^{20}$. Die Namen der Krankheit und ihre Bedeutungen lassen sich nach V. Klingmüller grob in sechs Gruppen aufspalten, wobei notwendige Korrekturen moderner medizingeschichtlicher Forschungen zum Thema eingebracht werden.

Die erste Gruppe nennt die Krankheit nach den Symptomen, wie Schorf (ahdt.), Horn (frk.) ${ }^{21}$, schwärende Haut (got.), die Glieder austrocknende Krankheit (arab.), Warzenausschlag (japan.), Schuppe (griech.) oder Taubheit (chin.);

die zweite Gruppe orientiert sich am Aussehen der Kranken bzw. den damit verbundenen Empfindungen für Außenstehende. So heißt die Krankheit im Isländischen «der stinkenden Leiche ähnlich». Als «Räuber» empfindet sie das Phönizische; «Elephantiasis» bzw. «Leontiasis» nennt sie das Griechische.

Geographische Lokalisierungsversuche hinsichtlich vermeintlicher Ursprungsländer der Krankheit sind vielfach zeitgebunden: elephantiasis graecorum, lepra arabum im Gegensatz zur lepra graecorum, die nicht der Lepradiagnose im modernen Sinn entspricht, und morbus phoenicicus nennt sie der Lateiner, Krimkrankheit der Uralrusse und Chinesenübel die Bewohner Hawaiis.

Nach dem Aufenthaltsort der Erkrankten nennt man die Krankheit bzw. die Kranken Aussätzige, Ausmärkige, Feld- und Sondersieche im Deutschen, Griechischen und Holländischen; Spitalseuche hingegen in den skandinavischen Sprachen (dänisch, norwegisch, schwedisch, estnisch). Religiöse Motive kommen zum Tragen im Französischen, wo Lepra als die Krankheit des Lazarus bekannt ist. «Von Gott geschlagen und geprüft» bzw. «vom Himmel als Strafe auferlegt» bedeutet der Wortinhalt im Hebräischen, Arabischen und Japanischen. In Ungarn bezeichnet man sie als Höllenkrankheit. Miselsucht oder Gute-Leute-Krankheit, Misellerie oder Kummer wird als Wortbedeutung im Deutschen, Französischen und Russischen angetroffen. Hier ist mehr die persönliche Situation des Erkrankten in den Vordergrund gerückt.

Aus dieser Vielzahl von Namensbedeutungen, von denen jede einem klar umrissenen Vorstellungsbild entspricht, das in der jeweiligen Gesellschaft lebendig war und mehr oder weniger bewußt gehalten wurde, werden nun die herausgegriffen, die zur Analyse derjenigen Weiheriten von Belang sind, mit deren Hilfe die Kranken zwar aus der Gesellschaft ausgeschlossen, keines- 
wegs aber aus dem gesellschaftlichen Bewußtsein getilgt wurden. Hier wurde ihr Gedächtnis vielmehr intensiviert, um dadurch der Bewältigung der zahlreich anfallenden sozial-karitativen Aufgaben am Leprakranken dienstbar gemacht zu werden ${ }^{22}$. Da die hier zu behandelnden Sequestrationsriten ${ }^{23}$ ausschließlich aus dem französischen Raum stammen, von wo aus sie in die westdeutschen und schweizerischen Grenzgebiete ausstrahlten, im gesamten übrigen deutschsprachigen Raum aber liturgische Quellen mit dem Ziel der Ausgesellschaftung Leprakranker nicht bekannt sind ${ }^{24}$, kommen für diese Untersuchung vorrangig die französischen, dann auch die deutschen Wortbedeutungen der Krankheit und die daraus ableitbaren Vorstellungsbilder im öffentlichen Bewußtsein in Betracht.

Das Fränkisch-Französische kennt das religiös motivierte «mal ladre» und die auf die Situation des Erkrankten bezogene Bezeichnung «misellerie». Für die Wirksamkeit auf das öffentliche Bewußtsein sind diese beiden Bedeutungen von Interesse: misellerie mit dem Grundgehalt elend, zum Erbarmen. Dies aktiviert einmal zum tätigen Helfen, drückt aber auch die abgesonderte und gemeinschaftsferne Lebensweise des Betroffenen aus. Der Gesichtspunkt des Erbarmens kommt verstärkt in der Wortbedeutung von mal ladre zum Ausdruck. Aus der Liturgie und insbesondere aus der Bildkatechese an Hand von Fresken an den Wänden romanischer Kirchen war jedem Menschen das Bild des Lazarus lebendig ${ }^{25}$. Sein Kontakt mit dem Haus und seinen Bewohnern war reduziert auf ein kaum mehr unterschreitbares Minimum und trotzdem nicht völlig aufgehoben. Lazarus wurde im biblischen Gleichnis wenigstens mit Abfällen versorgt und konnte darin die Nähe einer Gemeinschaft verspüren, die ihn zwar weitgehend isoliert aber nicht völlig ausgeschlossen und vergessen hatte.

Wesentlich andere Vorstellungsgehalte ergeben sich aus den Krankheitsnamen im deutschsprachigen Raum. Hier dominieren die Bezeichnungen, die den Aufenthaltsort und die Situation des Kranken ansprechen. Miselsucht, dem das lateinische miser zugrundeliegt, erweckt hier die Vorstellung von Elend, d.h. im unbewohnten, öden Land lebend. Vollends wird diese Bedeutung angesprochen in den Bezeichnungen aussätzig, ausmärkig, feldsiech, sondersiech. Die Trennung von der Gemeinschaft war hier keine partikuläre mehr wie im französischen Verständnis vom armen Lazarus, sondern war total vollzogen. Der am Aussatz Erkrankte im deutschen Sprachbereich durfte nicht an den Lazarus vor der Türe denken, der noch losen Kontakt mit der Gesellschaft hatte. Für ihn bedeutete die Krankheit das Ausgesetztsein, das Leben im unbewohnten Land, gelöst von jeglicher 
Gemeinschaft mit den Menschen. Damit soll indes nicht gesagt sein, daß all diese verschiedenen Wortinhalte zu jeder Zeit in gleich hohem Maße gültig und bewußt gewesen sind und das darin Ausgedrückte tatsächlich auch in die Praxis übergeführt worden ist. Es genügte aber offensichtlich, wenn sie als halbbewußte Tabuvorstellungen in der gelebten, gesprochenen und das Leben gestaltenden Sprache wachgehalten und beachtet wurden. Vielleicht ist dieser Sachverhalt auch ein erklärender Hinweis darauf, weshalb im deutschen Sprachraum, bis auf die Grenzgebiete zu Frankreich hin, kein Hinweis auf das Vorhandensein von Ritualien gegeben ist, die sich mit dem Ausschluß Leprakranker aus der Gesellschaft zu befassen gehabt hätten.

Der älteste erhaltene Ordo «Ad separandum leprosorum» ${ }^{26}$ stammt aus dem Kloster St. Albuin in Angers. Martène, sein Herausgeber, legt dessen Entstehungszeit in die Mitte des 11. Jahrhunderts; aus dieser Zeit stammt auch der Kodex, in welchem er aufgefunden wurde ${ }^{27}$. Mit dem darin ausgesprochenen Verbot, beim Ausschlußverfahren die Totenmesse zu feiern, dem Kranken das Totengewand anzulegen oder ihn wie bei den Exsequien zwischen brennenden Kerzen zu postieren, wird der Kranke als Kranker gesehen und nicht als für die Gesellschaft Toter. Als Formular für die Sequestrationsmesse soll die Kranken-, Sonntags- oder Heiliggeistmesse Verwendung finden; der Auszuschließende wird «Lazarus» genannt, d.h. in Gegenwart der mitfeiernden Gemeinde mit dem biblischen Lazarus identifiziert $^{28}$. Unter jeweiligen Segnungen werden dem in Prozession in die Kirche eingeholten Kranken - nach einer längeren eindringlichen Belehrung über seine nunmehrigen Pflichten ${ }^{29}$ - die Abzeichen seines neuen Standes übergeben: der Leprosenrock, das Trinkfäßchen, der Ledersack und die Holzklapper. Ferner wird er mit dem benötigten Haus- und Arbeitsgerät ausgestattet ${ }^{30}$. Am Ende der Zeremonie hat der Kranke einen neuen gesellschaftlichen und religiösen Status erlangt. Er ist zum Geweihten geworden und damit eo ipso aus der übrigen Gesellschaft herausgehoben; bezeichnenderweise ist die Einweisung ins Leprosorium dem Ritual der Aufnahme von Novizen ins Kloster und der Mönchsweihe nachgebildet ${ }^{31}$.

Der Ordo II im sehr jungen Reimser Rituale von $1585^{32}$ hebt hervor, daß beim Ausschluß nicht das Meßformular «Os justi» der Bekennermesse genommen werden solle, obgleich dies an einigen Orten noch üblich ist. Aus diesem Hinweis läßt sich erkennen: wurde für die Sequestrationsmesse das Meßformular für Bekennerheilige verwendet, dann war der Kranke, für den die Messe gefeiert wurde, direkt oder indirekt dem Heiligentyp des Bekenners gleichgestellt, zumindest angenähert. Dies soll nunmehr vermieden 
werden, denn mit dieser Angleichung an das Heiligenideal ist ein theologisches Problem aufgetreten. Es wird nämlich die Krankheit mit dem Fegefeuer gleichgesetzt, das jeder Gläubige erst nach seinem Tode zur Läuterung durchzustehen hatte, ehe er in die volle Gemeinschaft der Heiligen aufgenommen werden konnte ${ }^{33}$. Dem an Lepra Erkrankten blieb dieser jenseitige Läuterungsvorgang erspart, weil er durch die Krankheit ins Diesseits verlagert worden ist. Mit seinem Tode also tritt der Leprakranke unmittelbar in jenen Zustand der visio beatifica ein, der dem mittelalterlichen Menschen Lebenssinn und -ziel war. Damit glaubte man allem Anschein nach den Ausschluß des Kranken aus der menschlichen Gesellschaft genügend aufgewogen, und da dieser in dem Bewußtsein leben konnte, im Tode einem Heiligen gleichgestellt zu sein, mochte der Ausschluß für ihn die Trostlosigkeit verlieren, die er gemeinhin an sich trug. Das religiöse Tabu einer Weihehandlung gab nach Auffassung des Ordo II dem Ausgemeinschafteten hinreichenden Schutz. Es konnte den Kranken, von dessen Bewußtseinsdeformierung und seelischer Veränderung man bereits wußte ${ }^{34}$, davor bewahren, aus Angst oder Verbitterung zum Asozialen zu werden. Vielleicht spielt der reformatorische Angriff auf die Heiligenverehrung mit eine Rolle, weshalb man sich in diesem Ordo von der Vorstellung distanzierte, der Lepröse habe in seiner Krankheit das Fegefeuer auf Erden und sei im Tode ein Heiliger; möglicherweise setzte sich auch die Erkenntnis durch, daß im Leprosorium jahrhundertelang auch Haut- und Geschlechtskranke als Gottesleute behandelt wurden ${ }^{35}$.

Die auf diese Weise vor dem Kranken geschützte Gesellschaft der Gesunden war dem geweihten Kranken gegenüber zu besonderen Anstrengungen verpflichtet. Einmal im Jahr, in der Karwoche, durften die Leprösen aus ihrer Isolierung heraustreten und als Sinnbild der aus ihren Gräbern auferstandenen Toten die gesunden Menschen an Tod und Auferstehung erinnern ${ }^{36}$, was ihnen durch reichliches Almosen entgolten ward.

Einen ganz anderen Ton schlagen die jungen, aber noch vortridentinischen Ordines von Bourges/Sens und Châlon-sur-Saône ${ }^{37}$ an. Was in den Ordines von Angers und Reims untersagt ist, tritt hier in den Rubriken erdrückend in den Vordergrund. Die Krankheit und der krankheitsbedingte Ausschluß aus der Gesellschaft wurden, wie schon im langobardischen Recht, mit der gesellschaftlichen Toterklärung des Kranken belegt ${ }^{38}$. Das Motiv, der Gesellschaft gegenüber tot zu sein und damit alle Rechte in ihr verwirkt zu haben, spiegelt sich handgreiflich in diesen Sequestrationsriten wider; der Kranke ist für die Welt gestorben; was ihm bleibt, ist das Leben in 
Gott. Das Totengewand, mit dem er bekleidet wurde; die Messe mit anschließendem Libera; das symbolische Bewerfen mit Friedhofserde aus dem Begräbnisritus und die schwarzen Leichentücher, mit denen der Kranke bedeckt werden mußte, sorgten für die Eindeutigkeit und Unwiderruflichkeit des bevorstehenden Ausschlusses, im Zuge dessen auch das Vermögen des «Pauper», wie er nun genannt wird, eingezogen wurde, um nicht die Pfarrei als Träger des Leprosoriums unnötig zu belasten. Der abschließende Satz «Et si ainsi le faites, vous accomplirez votre purgatoire en ce monde, au partement duquel irez en paradis sans passer en purgatoire» hat nach diesem Ritus kaum mehr etwas Tröstliches an sich.

Das 1524 neu herausgegebene Rituale von Amiens sieht im Anschluß an ältere Ordines sogar vor, daß der Kranke in eine eigens zu diesem Zweck auf dem Friedhof ausgehobene Grube gelegt und vom Priester dreimal mit Erde beworfen wurde unter den Worten «De terra plasmasti me Domine ...» ${ }^{39}$. Immerhin aber läßt dieser unglaublich harte Ritus beim Begräbnis eines Ausgeschlossenen die Messe «Os justi», d.h. das Formular für einen nichtbischöflichen Bekennerheiligen zu und gibt damit wenigstens den Umstehenden die gläubige Gewähr, daß der Verstorbene mit seinem Tode unmittelbar in die Reihe der Heiligen aufgenommen worden ist.

An das skizzenhaft ausgebreitete Material, das im wesentlichen zwei Typen von liturgischen Ausschlußverfahren erkennen ließ, knüpft sich eine Reihe von Fragen und Vermutungen, die einer Klärung bedürfen und vom Hintergrund der gesellschaftlichen Veränderungen im Verlauf mehrerer Jahrhunderte her zu deuten sind. Zeitliche Fixierungen sind nicht möglich, da im kirchlich-kultischen Bereich den veränderten Umständen erst dann verbindlich Rechnung getragen werden konnte, als diese $\mathbb{W}$ andlungen einen gewissen Abschluß erreicht und die neuen Verhältnisse sich einigermaßen konsolidiert hatten. Zwei Verlagerungen im öffentlichen Bewußtsein lassen sich feststellen, die in den liturgischen Quellen ihren Niederschlag gefunden haben. Die erste darf im Zusammenhang mit dem gesellschaftlichen Aufbruch am Beginn des 11. Jahrhunderts und den dadurch ausgelösten Migrationen gesehen werden; eine Zeit also, in der die Lepra und alles, was dafür gehalten wurde, zu einer Massenkrankheit größeren Ausmaßes geworden sein konnte. Zahlreiche Städteneugründungen neben älteren aufstrebenden Zentren von Handel und Verkehr wurden zu bisher nicht gekannten Ballungsräumen mit starker Fluktuation, in denen sich die Lepra und mit ihr gleichgesetzte kontagiöse Erkrankungen rasch verbreiten konnten. Kirche und Gesellschaft waren gleichermaßen angesprochen, das Problem 
zu bewältigen und die Krankheit durch verschärfte Bekämpfungsmaßnahmen in den Griff zu bekommen ${ }^{40}$.

Die zweite Bewußtseinswende liegt im 15./16. Jahrhundert und findet in der gesellschaftlichen Toterklärung der Leprösen in den zu Beginn des 16. Jahrhunderts neu redigierten Ritualien zumindest ihren theoretischen Höhepunkt und zugleich Abschluß ${ }^{41}$. Es ist zu fragen, welche geistigreligiösen, politisch-gesellschaftlichen oder wirtschaftlichen Faktoren, aber auch welche Erkenntnisse der medizinischen Forschung diese Bewußtseinsänderungen ausgelöst haben können ${ }^{42}$. Daß die Bischöfe im spanischgallischen Raum schon früh die Leprakranken in ihren Schutz nahmen und Bestimmungen zu ihrer Isolierung von Gesunden erließen, geht einmal auf das biblische Vorbild der priesterlichen Lepraschau zurück ${ }^{43}$ und ist zum anderen erklärbar aus der Wahrnehmung herrschaftlich-administrativer Funktionen der Kirche in der spätantik-frühmittelalterlichen Stadt ${ }^{44}$. Der religiöse Tabucharakter war bei ihren Verordnungen noch nicht dominierend, vielmehr stand die Sorge um den Kranken im Vordergrund des Bemühens. Jahrhunderte später war das religiöse Tabu vorherrschend, als die Leprösen durch einen der Mönchsweihe nachgebildeten Ritus von der Gesellschaft abgetrennt werden mußten.

Mit der Quasi-Weihe der Sequestration war ein neuer gesellschaftlicher wie religiöser Status erreicht, der sich auch in der Benennung der Leprösen niedergeschlagen hat ${ }^{45}$. Damit war der Ausschluß aus der Gesellschaft irreversibel geworden und hatte öffentlich-rechtlichen Charakter bekommen ${ }^{46}$. Ein Ausbruch aus dem religiösen Tabu, d.h. die Rückkehr in die volle menschliche Gemeinschaft über das Maß des Zugestandenen hinaus, hätte diesen als gottgegeben angesehenen Status verletzt und schwerste Strafen nach sich gezogen ${ }^{47}$.

Es ist hier die Frage aufzuwerfen, welche Schichten der mittelalterlichen Gesellschaft von der Lepra befallen waren und wer davon die Möglichkeit gehabt hat, sich einem nicht tabuisierten Isolierungsgebot der kirchlichen oder weltlichen Obrigkeit zu widersetzen.

Der erste Teil der Frage ist rasch zu beantworten: seit der Zeit der Kreuzzüge waren es nicht mehr nur die untersten Volksschichten, die an Lepra und lepraähnlichen Dermatosen erkrankten, sondern auch die höheren Stände des Adels und Klerus ${ }^{48}$. Die im 11. Jahrhundert einsetzende Errichtung von Leprosorien in allen größeren Städten, die sich bis ins 14. Jahrhundert auf dem breiten Land fortsetzte ${ }^{49}$, legt die Vermutung nahe, daß den leprösen Angehörigen höherer Gesellschaftsschichten nicht 
mehr das Los des Lazarus vor der Türe der Gesunden oder gar das der ausmärkigen Feldsiechen zugemutet wurde. Sie standesgemäß unterzubringen könnte ein Grund dafür gewesen sein, daß zahlreiche gut dotierte Stiftungen für Lepröse entstanden sind, in denen noch weit hinein ins Spätmittelalter adelige und geistliche Leprosen nachweisbar sind ${ }^{50}$. Von diesen Stiftungen profitierten wohl auch ärmere Kranke, denen vor Errichtung der zahlreichen Leprosorien das harte Leben außerhalb der Gesellschaft bevorstand. Diejenigen Schichten der mittelalterlichen Gesellschaft aber, für die vermutlich die gut dotierten Leprosorien gestiftet worden waren, die Potentes nämlich, waren es aber, die durch ihren Geburts- oder Berufsstand über Möglichkeiten und auch die nötige Macht verfügt haben, den obrigkeitlichen Ausschließungsverordnungen zu widerstehen oder sich ihnen sonst erfolgreich zu entziehen. Ein Brief Alexanders III. an den Bischof von Lincoln aus dem Jahre 1183 oder 1184 bestimmte, daß einem an Lepra erkrankten geistlichen Pfründeinhaber wenigstens ein Koadjutor beizugeben sei, um ein scandalum unter den Gläubigen zu verhindern ${ }^{51}$. Aufgrund seiner Rechtsstellung war dieser nämlich nicht ohne weiteres von seiner Pfründe amovibel und konnte sich einem nur administrativen Ausschlußverfahren mit Erfolg entgegenstellen ${ }^{52}$. Die Schaffung eines eigenen kirchlichen Status jedoch, der dem Status des Bekennerheiligen ähnlich war und eo ipso von den übrigen Menschen absonderte, könnte notwendig geworden sein, um die Gesellschaft wirksam vor der Krankheit zu schützen und vor allem standesbedingte Rücksichten gegen höhergestellte Leprosen dadurch zu umgehen, daß die Erlangung der visio beatifica unmittelbar im Tode als Ziel vor Augen gestellt wurde. Dies war von der Person und ihrer Stellung innerhalb der geburts- wie berufsständisch aufgebauten Gesellschaftsordnung unabhängig.

Schwieriger gestaltet sich der Erklärungsversuch, warum in der vorreformatorischen Zeit mit der wiederaufgegriffenen Toterklärung ${ }^{53}$ des Leprakranken in das geschlossene und noch intakte religiöse Tabu Elemente des bürgerlichen Rechtes (Besitz- und Erbunfähigkeit) eindringen konnten. Wir haben gesehen, daß Lepra kein Grund war, bepfründete Ämter zu verlieren oder ein bestehendes Eheband zu annullieren. Auch der lepröse König Balduin von Jerusalem hat, wenn wir Burchard von Ursberg Glauben schenken dürfen, sich freiwillig der Ehe enthalten und ungezwungen seinen Neffen Wilhelm zum Nachfolger gekrönt ${ }^{54}$. Die persönliche Freiheit des Erkrankten war zwar eingeschränkt, er war aber durch seine Krankheit noch keineswegs zum Pauper im rechtlich-politisch-öffentlichen Sinne geworden. 
Auch spricht die Zwangseinweisung ins Leprosorium nach dem Ordo I nicht vom Güterverlust des Eingewiesenen, obwohl anzunehmen ist, daß die einer klösterlichen Kommunität angeglichene Lebensform im Leprosorium den Gebrauch des Privateigentums beschnitt. In diesem Zusammenhang bemerkenswert und auf eine mögliche Antwort hinweisend, erscheint eine Stelle im Defensor pacis des Marsilius von Padua ${ }^{55}$. Dessen Auswirkungen auf die staatskirchlichen Anschauungen insbesondere der Reformationszeit sind hinreichend bekannt ${ }^{56}$. Besagte Stelle ${ }^{57}$ ordnet die Leprosenschau und die Feststellbarkeit der Krankheit allein der Autorität des Arztes zu, der seine Legitimation ausschließlich vom weltlichen Gesetz erhalten hat. Verleumdungen hingegen nennt er die Aussagen und Anordnungen der Hl. Schrift zu diesem Thema und meint damit die Leprosenschau der Priester im mosaischen Gesetz und im Anschluß daran die kirchliche Leprosenschau ${ }^{58}$. Damit entkleidet Marsilius die Krankheit ihrer Sakralität. Mag sie auch GottesgeiBel sein, als Krankheit unterliegt sie dem fachlichen Urteil des Arztes und nicht dem des Theologen. Damit aber war von der Krankheit nicht nur der Gottesfluch genommen, sondern auch der Gottesschutz, der ihrer Sakralität anhaftete. Sie war Gegenstand der gesellschaftlich-rechtlichen Sphäre des Staates geworden und somit dessen Normen und Prinzipien unterworfen.

$\mathrm{Da} ß$ diese Entwicklung nicht erst in der Reformationszeit einsetzt, zeigt einmal das allmähliche Verschwinden zahlreicher Leprosenhäuser in den Städten, bzw. deren Umwandlung in Spitäler, und zwar nicht mehr unter kirchlicher, sondern unter städtischer Obhut. Dies läßt sich seit dem 13. Jahrhundert beobachten ${ }^{59}$. Dem widerspricht nicht die nachweisliche Stiftungsfreudigkeit für neue Leprosorien noch im 15. und 16. Jahrhundert vor allem auf dem flachen Land ${ }^{60}$. Als weiteres Indiz kann die Umwandlung des Lazaritenordens zunächst in einen Ritterorden im 13. Jahrhundert und dessen Aufhebung durch den Papst 1490 gesehen werden ${ }^{61}$. Die Wende zeichnet sich ferner ab in einer Verfolgungswelle gegen Leprosen, die den Judenpogromen parallel läuft und, wie allgemein angenommen wird, auf den reichen Besitz der Leprosorien abzielt. Benedikt XII. droht für den Fall der Verfolgung von Leprosen die Exkommunikation an, woraus doch geschlossen werden darf, daß diese Bewegung nicht lokal beschränkt war ${ }^{62}$; inwieweit diese erneut sakral tabuisierende Maßnahme von Erfolg begleitet war, entzieht sich unserer Kenntnis.

Dabei verträgt es sich noch mit der gläubig-erregten, apokalyptisch gefärbten spätmittelalterlichen Religiosität, daß trotz der fortschreitenden Entsakralisierung der Krankheit, der zunehmenden Erkenntnis, daß jahr- 
hundertelang unter dem Gottesschutz des Leprosoriums mehr Nicht-Lepröse gut gelebt haben, auch weiterhin in den Kartagen die Bewohner der Leprosenheime bettelnd durch die Städte ziehen ${ }^{63}$, wo sie als lebendige Zeichen der erwarteten Auferstehung, als die aus ihren Gräbern Erstandenen der Endzeit angesehen wurden. Fraglich bleibt es allerdings, und ein Kenner der Materie ${ }^{64}$ bezweifelt dies, ob die ins 16. Jahrhundert tradierten und teilweise noch neu kodifizierten Sequestrationsriten in dieser Zeit überhaupt noch Anwendung gefunden haben und nicht lediglich als ehrwürdige historische Relikte sich in den liturgischen Büchern erhalten haben.

\section{Anmerkungen}

${ }^{1}$ Der Beitrag wurde gesendet am 26. Januar 1975; vgl. auch das Buch der Autorin, Dem Tode geweiht? Lepra ist heilbar, Percha 1974.

${ }^{2} \mathrm{Zu}$ dieser Problematik siehe die grundlegenden Ausführungen von G. W. Allport, Die Natur des Vorurteils, Köln 1971.

${ }^{3} \mathrm{Ob}$ in der verstärkten Auseinandersetzung mit dem Thema Krankheit und Tod in jüngster Zeit bereits eine Tendenzwende zu sehen ist, mag dahingestellt bleiben. Vgl. auswahlweise Ph. Ariès, Geschichte des Todes. Abendländische Einstellungen zum Tode vom 9. Jahrhundert bis zur Gegenwart. München 1979; F.Böckle, Menschenwürdig sterben, Einsiedeln/ Köln 1979; A. Gügler (Hrsg.), Wenn Kinder nach dem Tod fragen, München 1979; A. Mander, Kunst des Sterbens, Regensburg ${ }^{5}$ 1979; J. Mayer-Scheu, R. Kautzky, Heilen - die vergessene Dimension des Krankenhauses. Freiburg/Göttingen 1979; G. Scherer, Das Problem des Todes in der Philosophie, Düsseldorf 1979; P.Tournier, Vom Sinn unserer Krankheit, Freiburg/Basel/Wien 1979.

${ }^{4}$ Nach Aretaios, der an der Wende vom 2. ins 3. nachchristliche Jahrhundert lebte und die eindrücklichste, ja klassische Beschreibung der Elephantiasis lieferte, wurden bei Griechen und Römern die erkrankten Personen ausgemeinschaftet. Vgl. H. M. Koelbing und A. Stettler-Schär, Aussatz, Lepra, Elephantiasis Graecorum - zur Geschichte der Lepra im Altertum. In: H.M. Koelbing/M.Schär-Send/A.Stettler-Schär/H.Trümpy, Beiträge zur Geschichte der Lepra. Zürcher medizingeschichtliche Abhandlungen, Neue Reihe 93, Zürich 1972, 34-54; hier 43ff., ferner A. Stettler, Lepra und Pest in der Antike. In: Antike Welt 8/4, Zürich 1977, 42-44, hier 43.

${ }^{5}$ Es ist in keinem Falle der Nachweis zu erbringen, daß vorhandene Sequestrierungs-Riten auch tatsächlich zu jeder Zeit in Anwendung gebracht worden sind.

${ }^{6} \mathrm{Vgl}$. hierzu die wenig differenzierende und zu historischen Verallgemeinerungen neigende Untersuchung von O.Schlotter, Die Geschichte der Lepra und Pocken in Europa, München $1966,11 \mathrm{ff}$.

7 Vgl. hierzu die ältere Studie von G. Kurth, La Lèpre en Occident avant les Croisades, Paris 1891.

${ }^{8}$ MGH Conc. I, Cap. XXI, p. 107/15, Concilium Aurelianense, 28.10.549: «... specialiter tamen de leprosis id pietatis causa convenit, ut unusquisque episcoporum, quos incolas hanc 
infirmitatem incurrisse tam territorii sui quam civitatis agnoverit, de domo ecclesiae iuxta possibilitatem victui et vestitui necessaria subministret ...»

MGH Conc. I, Cap. VI, p. 154/27, Concilium Lugdunense, Mai 583: «... ut uniuscuiusque civitatis leprosi ... ab episcopo ecclesiae ipsius sufficientia alimenta et necessaria vestimenta accipiant, ut illis per alias civitates vagandi licentia denegetur».

Vgl. ferner F. Merzbacher, Die Leprosen im alten kanonischen Recht. In: ZSavRGkan 84 (1967), 27-45; hier 28.

${ }^{9}$ MGH Leg. II, Capitularia, Cap. XX, p. 244: «De leprosis, ut se non intermisceant alio populo».

10 MGH Script. rer. Merov. I, Gregorii ep. Turon. hist. liber VIII, 402/1; ebd., Eugipii Vita S. Severini, 26/1, 34/2.

MGH Script.rer. Merov. III, Vita vel passio S. Desiderii a Sisebuto Rege composita, 632/14; ebd., Passio S. Desiderii Episc. et Mart., 640/10; ebd. Vita Sti. Gangerici Ep. et Conf. 653/20. MGH Script. rer. Merov. IV, Vitae Columbani Abbatis II, 147/7; ebd. Vita Romarici Abbatis Habendensis, 223/19; ebd., Vita Richarii Conf. Centulensis Auctore Alcuino, 392/11; ebd., Virtutes Sti. Fursei, 441/6.

Ferner MGH Script. rer. Merov. V, 449/10, 565/6, 620/20; VI, 383/28; VII, 446/5.

11 Weitere Beispiele von Heilungen führt F.Meffert, Caritas und Volksepidemien, Freiburg 1925, 37 ff., an; zur Gesamtthematik des Heiligen in der merowingischen Gesellschaft siehe das grundlegende Werk von F. Graus, Volk, Herrscher und Heiliger im Reich der Merowinger. Studien zur Hagiographie der Merowingerzeit. Prag 1965; ferner hat zu diesem Thema Stellung genommen K. Bosl, Der «Adelsheilige». Idealtypus und Wirklichkeit, Gesellschaft und Kultur im merowingerzeitlichen Bayern des 7. und 8. Jahrhunderts. Gesellschaftsgeschichtliche Beiträge zu den Viten der bayerischen Stammesheiligen Emmeram, Rupert und Korbinian. In: Speculum Historiale. Festschrift J.Spörl, München 1965, 167-187.

12 Auf diesen Umstand weist mit Nachdruck hin A.Stettler-Schär, Leprologie im Mittelalter und in der frühen Neuzeit, in: H.M.Koelbing/M.Schär-Send/A.Stettler-Schär/H.Trümpy, Beiträge zur Geschichte der Lepra, a. a.0., 55-83, hier 66; ferner in «Lepra und Pest in der Antike», a.a.O., 43.

13 a Zu den Kreuzzügen vgl. S. Runciman, Geschichte der Kreuzzüge, München 1968.

13b $\mathrm{Zu}$ dieser Problematik siehe den bereits genannten Beitrag «Aussatz, Lepra, Elephantiasis Graecorum ...» von H.M. Koelbing und A.Stettler-Schär, a.a.O. Zunehmende diagnostische Sicherheit in der Behandlung der lepromatösen wie tuberkuloiden Form der Krankheit ist erst seit dem ausgehenden 13. Jahrhundert feststellbar; vgl. hierzu A. Stettler-Schär, Leprologie ..., a. a. O., bes. $66 \mathrm{ff}$.

${ }^{14}$ Diese Meinung vertritt, ohne die Lepra von anderen Hautläsionen abzugrenzen, auch noch O. Schlotter, Die Geschichte der Lepra und Pocken in Europa, München 1966, 2.

${ }^{15}$ Siehe auswahlweise K.Schneider, Die Geschichte der Lepra im Kreise Memel und das Lepraheim in Memel, Berlin 1942; A. Deibele, Das Katharinenspital zu den Sondersiechen in Schwäbisch Gmünd, Schwäbisch Gmünd 1966; J.G.Andersen, Studies in the medieval diagnosis of Leprosy in Denmark, Copenhagen 1969; G. H. Klövekorn, Der Aussatz in Köln, München 1972; ausschließlich auf Sekundärliteratur bauen die Ausführungen O. Schlotters zum Thema der Ausbreitung der Lepra im europäischen Raum auf.

${ }^{16}$ MGH Dipl. Karol. I, 259/40, Immunitätsbestätigung Karls d.Gr. an die Kirche von Paris (774-800): «Si quis vero, quod absit, contra hoc nostrae auctoritatis edictum vel concessum 
insurgere temptaverit, in dei omnipotentis incurrat maledictionem et artatus atque constrictus igneis cathenis profunde inferni percussione sanctae dei genetricis Mariae et omnium sanctorum horum episcoporum sit anathematizatus atque divina ultione perfossus animum exspiret vindicationis lepre laniatus.»

Siehe ferner Mansi, Suppl. ad Tom. XVII, 1097, Liber sept. Capitularium, c. CCCXXI; ebd., 1293, Capitula Herardi Archiep. Turon., wo dieses Zitat wiederholt wird.

17 Vgl. P. A. Dold, Zum Langobardengesetz. Neue Bruchstücke der ältesten Handschrift des Edictus Rothari. In: Deutsches Archiv für Geschichte des Mittelalters 4 (1941), 1-52.

MGH Fontes iur. Germ. antiqui, c. 176/36, Edictus Rothari; ebd., c.180/37.

${ }_{18}$ MGH Leg. II, Capitularia, Decretum Compendiense 757, 39/11; ebenso bei Mansi, Suppl. ad Tom. XVII, 184, Cap. Compendiense, 787, c. XVI.

${ }^{19}$ Papst Alexander II. (1061-1073), vor allem aber Alexander III. (1159-1181), dann auch Urban III. (1185-1187) wandten sich strikt gegen die Möglichkeit einer Eheauflösung im Erkrankungsfall eines Ehepartners. Vgl. Ph. Jaffé, Regesta Pontificum Romanorum II, 370, n. 13773 (8848), Schreiben «Leprosis autem» Alexanders III. an den Bischof von Beauvais, worin er hervorhebt, «mulierem lepra percussam seu alia gravi infirmitate detentam non esse a viro propterea separandam ...» Zur Stellung des Eherechtes der Leprosen innerhalb der kanonistischen Entwicklung siehe vor allem F. Merzbacher, Leprosen ..., a. a. O., bes. $32 \mathrm{ff}$.

${ }^{20}$ Die Namen für die Lepra sind entnommen V. Klingmüller, Die Lepra, Berlin 1930, 2 f.; sie sind jedoch zu ergänzen und teils zu berichtigen durch H.M.Koelbing und A.StettlerSchär, Aussatz, Lepra ..., a. a. O. Ohne auf die Einzelbeiträge näher eingehen zu können, sei auf den für diese Thematik einschlägigen Sammelband von L.Schmidt, Wortfeldforschung. Zur Geschichte und Theorie des sprachlichen Feldes. Darmstadt 1973, verwiesen.

${ }^{21}$ Nach Trümpy bezieht sich der Begriff «Horn» jedoch nicht auf ein Krankheitssymptom, sondern auf das Hornsignal zur Warnung Gesunder. Vgl. H.Trümpy, Die Aussätzigen in der mittelalterlichen Gesellschaft. In: Beiträge zur Geschichte der Lepra, a. a. O., 84-93, hier 84 .

${ }^{22}$ Schon Gregor II. hatte in seinem Schreiben vom 22.11.726 an Bonifatius das Recht der Leprösen auf Teilhabe an der Eucharistiefeier betont: MGH Epist. III, 277/1, weiterhin Papst Zacharias in seinem Brief an Bonifatius vom 4.11.751: MGH Epist. III, $370 / 41$.

${ }^{23}$ Die Riten sind veröffentlicht bei E. Martène, De antiquis ecclesiae ritibus libri III, Antwerpen 1763, 358-362. Weitere Riten aus Metz (1543), Toul (1616) und Verdun (1507-1554), die bei Martène nicht enthalten sind, von den hier aufgeführten Riten jedoch wenig abweichen, bringt J.P. Kirch, Die Leprosorien Lothringens, insbesondere die Metzer Leproserie S.Ladre bei Montigny. In: Jahrbuch d. Gesellsch. f. lothr. Geschichte und Altertumskunde 15 (1903), 46-109, hier 67-74. Widersprochen werden muß dort der Verallgemeinerung S.66, wonach die Sequestrations-Zeremonien mit Ausnahme verschiedener Einzelheiten «im Großen und Ganzen dieselben in der ganzen Christenheit» gewesen seien. Zu dieser Behauptung fehlen die offensichtlich nicht vorhandenen Belege. Auf das Fehlen ähnlicher Riten für Deutschland weist auch F. Meffert, Caritas und Volksepidemien, a. a. O., 85, hin. Auch A.Franz, der profunde Kenner der deutschen liturgischen Quellen, führt sie in seinem zweibändigen Werk Die kirchlichen Benediktionen im Mittelalter, Graz ${ }^{2} 1960$, nicht auf. 
${ }^{24}$ Lediglich im Codex epistularis der Monumenta Garsensia (MB I, Nr.34, S. 100 f.) findet sich ein Briefvordruck der geistlichen Aufsichtsbehörde (Archidiakonat Gars) zur Bestätigung an die Pfarrämter, daß bei der untersuchten Person kein positiver Leprabefund vorliegt.

${ }^{25}$ Lukasevangelium 16, 19-31.

${ }^{26}$ Es handelt sich hier nicht um Weihen im streng kirchenrechtlichen Sinn, weshalb besser der unschöne Ausdruck «Quasi-Weihe» verwendet werden sollte. Vgl. E. Eichmann/K. Mörsdorf, Kirchenrecht II, München/Paderborn/Wien ${ }^{10} 1961,96 \mathrm{ff}$.

${ }^{27}$ E. Martène, a. a. O., XIX, Syllabus librorum ...: Die Kodifizierung des Ritus in der Mitte des 11. Jahrhunderts schließt nicht aus, daß er bereits längere Zeit vorher in Gebrauch gewesen ist.

${ }^{28}$ Martène, 358, Ordo I, Modus pro ejectione leprosorum: «... Primo presbyter indutus superpelliceo cum stola \& aqua benedicta accedat ad domum Lazari, qui Lazarus indutus tunica, seu clamyde lazareo debet venire ad ecclesiam cum sacerdote $\&$ processione, nec induitur pannis mortuorum ... quia non est mortuus corpore, sed propter morbum leprae ab omnibus est expulsus ...»

${ }^{29}$ Die wichtigste Verpflichtung ist das Meiden der Orte und Gelegenheiten zur Begegnung mit Gesunden, also der Kirchen, Jahrmärkte, Mühlen, Brücken, Wirtshäuser, überhaupt großer Menschenansammlungen; ferner das Verbot, Dinge anzufassen und zu benützen, mit denen Gesunde in Berührung kommen könnten (Geschirr, Brunnen, Kleider usw.).

${ }^{30}$ Es folgen die Gegenstände im Originalwortlaut des Ritus: «... Premier, une tarterelle, souillers, chauses, robe de camelin, une housse, \& un chaperon de camelin, deux paires de drapeaux, un baril, un entonnoir, une couroie, ung coustel, une escuelle de bois. Item on luy doit faire une maison \& ung puis; il doit avoir ung lit estofé de coutte, coussin \& couverture, deux paire de drap à lit, une hache, ou ung escrin fermant à clef, une table, une selle, une lumiere, une paelle, une aindier, des escuelles à mangier, ung bassin, ung pot à mettre cuire la chair.»

${ }^{31}$ Vgl. P.de Puniet, Das Römische Pontifikale II, Klosterneuburg 1935, 55 ff.; vgl. ferner F.Meffert, Caritas und Volksepidemien, a. a. O., 124ff., der das klosterähnliche Leben in den Leprosorien schildert. Bestätigt wird dies durch die sehr junge Hausordnung des St. Servatiusstiftes in Augsburg aus dem Jahre 1613. Das Stift war einstens als Leprosenhaus gegründet worden: Stadtarchiv Augsburg, Akt «Krankenhaus II», St. Servatiusstift Nr.13.

${ }^{32}$ Martène, 359 f.: Dieser Ordo - der von Martène vorangestellte Syllabus, XXII, nennt die Quellen dieses Rituale «vetus», d.h. mit Sicherheit vor dem 16. Jahrhundert entstanden enthält sämtliche Meßtexte, wobei die Lesungen auf den aussätzigen Syrer Naaman und die zehn Aussätzigen im 17. Kapitel des Lukasevangeliums eingehen.

${ }^{33}$ Klar ausgesprochen findet sich dieser Gedanke, allerdings in anderem Zusammenhang, in den noch zu besprechenden Ordines III und V.

${ }^{34} \mathrm{Vgl}$. S. N. Brody, The disease of the soul. Leprosy in medieval literature. London 1974; ferner W. Frohn, Lepradarstellungen in der Kunst des Rheinlandes, Berlin 1936.

${ }^{35}$ V.Klingmüller, Lepra, a.a.O., 11. A.Stettler-Schär, Leprologie, $76 \mathrm{ff}$., weist auf die kritische Auseinandersetzung der Ärzte des 16. Jahrhunderts mit den Autoren der Überlieferung hin, in denen Fehldiagnosen endgültig beseitigt werden können. Der Mediziner Felix Platter (1536-1614) sieht Syphilis und Skorbut bereits deutlich getrennt von der Lepra.

${ }^{36}$ Diesen Brauch erwähnen F.Meffert, Caritas und Volksepidemien, a.a.O., 82, ferner 
W. Rüger, Mittelalterliches Almosenwesen. Die Almosenordnungen der Reichsstadt Nürnberg. Nürnberg 1932, 24.

${ }^{37}$ Martène, $360 \mathrm{f}$., Ordo III aus dem Rituale von Bourges und Sens; Ordo V aus dem Rituale von Châlon-sur-Saône; da der Syllabus, XX, beide Ritualien mit «vetus» bezeichnet, darf ihre Entstehung wohl vor dem 16. Jahrhundert angenommen werden.

${ }^{38}$ Der Kranke wird in der Kirche so postiert und behandelt, wie es mit dem Leichnam beim Requiem geschieht, «... similitudinem mortui gerens, quamvis vivat in corpore»; mit den Worten «sis mortuus mundo, vivens iterum Deo» wird der Lepröse mit Erde beworfen.

39 «In meditullio temporis paretur parva fovea in cimiterio ad quam incedant cantando Resp. Libera me Domine \& c., cum versiculis, \& eam ingradiatur infirmus, \& sacerdos accipiat terram cum palla, quam trina vice ponat super caput infirmi, si laicus fuerit; si vero fuerit sacerdos, jacet terram super corpus ejus ...»

${ }^{40}$ Zur gesellschaftlichen Mobilität dieser Epoche siehe K.Bosl, Die Grundlagen der modernen Gesellschaft im Mittelalter, 2 Bde., Stuttgart1972, $161 \mathrm{ff} ., 212 \mathrm{ff}$.

${ }^{41}$ Auch in dieser Epoche stehen, wie schon im 11. Jahrhundert, weniger religiöse als vielmehr gesellschaftliche Umbrüche im Vordergrund. Pauschal darf gesagt werden, daß mit der Einführung der Reformation städtische Herrschaft und bürgerliches Selbstbewußtsein mit der Übernahme zahlreicher ehedem rein kirchlicher Kompetenzen ihren Höhepunkt erreicht hatten. Vgl. hierzu B. Moeller, Deutschland im Zeitalter der Reformation, Göttingen 1977, $31 \mathrm{ff} ., 42 \mathrm{ff}$., vor allem $111 \mathrm{ff}$.

${ }^{42}$ Hier ist auf die zusammenfassenden, auf das komplexe Wechselspiel aller in einer Gesellschaft wirkenden Kräfte sich beziehenden Studien von Karl Bosl zu verweisen: K.Bosl, Der Aufbruch von Mensch und Gesellschaft. Eine epochale Struktur in der europäischen Geschichte. In: R.Krohn/B.Thum/P. Wapnewski, Stauferzeit I. Geschichte, Literatur, Kunst, Stuttgart 1979, 11-27; ders., Die Reformation. Versuch einer Bestimmung ihres historischen Ortes und ihrer Funktion in Gesellschaft und Kultur Europas. In: ZBLG 31 (1968), 103-123; ders., Gesellschaftliche Anpassung und persönliches Engagement. Kirche, Welt und Individuum im Reformationszeitalter (1420-1530). In: A. Schade, Albrecht Dürer. Kunst einer Zeitwende. Regensburg 1971, 17-37. Zur diagnostischen Entwicklung in der Leprabehandlung seit dem 13.Jahrhundert siehe A.Stettler-Schär, Leprologie, a. a. O., 66-83.

${ }^{43}$ Siehe hierzu die Reinheitsbestimmungen im 13. und 14. Kapitel des dritten Mose-Buches, obgleich es inzwischen gesicherte Erkenntnis ist, daß die dort beschriebene weißfleckige Dermatose so wenig eine Lepradiagnose im modernen Sinne darstellt, wie die Krankheit des Hiob. Vgl. H.M. Koelbing und A.Stettler-Schär, Aussatz, Lepra, Elephantiasis Graecorum, a. a. O., $38 \mathrm{f}$.

${ }^{44}$ Vgl. K.Bosl, Grundlagen, a. a. O., $18 \mathrm{ff}$., mit zahlreichen Literaturhinweisen, ferner die von F.Prinz herausgegebene Aufsatzsammlung Mönchtum und Gesellschaft im Frühmittelalter, Darmstadt 1976.

${ }^{45}$ Sie werden Gute Leute, Pauperes, Martyres Christi genannt, ferner Lazari, Malozen, Gottesarme. Siehe hierzu G. Kurth, La Lèpre en Occident, a.a. O., 19; F. Meffert, Caritas, a. a.O., 137; T.Stark, Christliche Wohltätigkeit im Mittelalter ..., a.a. O., 54; A. Mitterwieser, Verzeichnis der in Bayern entstandenen städtischen und märktischen Hospitäler, Leprosenhäuser und verwandten Wohltätigkeitsanstalten. In: M. Doeberl/K.v. Reinhardstöttner, Forschungen zur Geschichte Bayerns 14 (1906), 289-314; hier 290 f., 293; R. Vir- 
chow, Zur Geschichte des Aussatzes und der Spitäler, besonders in Deutschland. In: Archiv f. pathol. Anatomie und Physiologie und für klin. Medizin 18 (1860), 273-329, hier 328.

${ }^{46}$ Vgl. hierzu die Ausführungen über das Weiherecht bei Eichmann/Mörsdorf, Kirchenrecht II, a. a. $0 ., 96 \mathrm{ff}$.

${ }^{47}$ Zur Tabuverletzung und zu den geglaubten Folgen siehe L. Lévy-Bruhl, Die geistige Welt der Primitiven, Darmstadt ${ }^{2} 1969,242$ ff., ferner S. Freud, Totem und Tabu, Frankfurt 1962, und die Einzelbeiträge in L.Petzold (Hrsg.), Magie und Religion, Darmstadt 1978.

${ }^{48}$ So F.Meffert, Caritas, 47; J.P.Kirch, La Lèpre, 54, 74f.; V.Klingmüller, Lepra, 55; T.Stark, Wohltätigkeit, 55; D. Knowles/R.N.Hadcock, Medieval Religious Houses. England and Wales. London/New York/Toronto 1953, 305.

${ }^{49}$ In Auswahl siehe F. J. R. Tolivar, Hospitales de leprosos en Asturia durante les edades media y moderna, Salamanca 1966; F. Bühler, Der Aussatz in der Schweiz, 3 Teile, Zürich 1902-1905; für Bayern einschließlich der Rheinpfalz siehe A. Mitterwieser, Geschichte der Stiftungen und des Stiftungsrechtes in Bayern, München 1907, $79 \mathrm{ff}$;; für Württemberg R.Virchow, Zur Geschichte des Aussatzes ..., $75 \mathrm{ff}$. Eine Liste aller vor dem 12. Jahrhundert in Europa entstandenen Leprosorien bringt G. Kurth, La Lèpre, 25 f.

${ }^{50}$ Auf die Existenz von Siechenhäusern für Arme und Reiche in Metz und Troyes weist H.Trümpy, Die Aussätzigen in der mittelalterlichen Gesellschaft, a. a. O., 89, ausdrücklich hin. Davon ausgehend wäre die naheliegende Frage zu überprüfen, ob auch in anderen Städten mit mehreren Leprosorien ständische Rücksichten bei deren Gründung maßgebend waren. Ferner müßten die Titelheiligen von Leprosorien und ihren Kirchen daraufhin überprüft werden, ob typische Standesheilige (z.B. Georg als Adelspatron) bei der Namengebung Berücksichtigung gefunden haben. Beide Fragestellungen übersteigen den Rahmen dieser Arbeit.

${ }^{51}$ Der Brief ist abgedruckt bei W.Holtzmann/E. W. Kemp, Papal Decretals Relating to the Diocese of Lincoln in the Twelfth Century, Hereford 1954, $52 \mathrm{f}$.

${ }^{52}$ Eingehender beschäftigt sich mit diesen Fragen F. Merzbacher, Leprosen, a. a. O., $40 \mathrm{ff}$.

${ }^{53}$ Die Rubriken zum Ordo I von Angers (11.Jh.) betonen, «quia non est mortuus corpore, sed propter morbum leprae ab omnibus est expulsus». Im Ordo III von Bourges/Sens (vor dem 15. Jh.) lauten die Rubriken schon «similitudinem mortui gerens, quamvis vivat corpore», und in Ordo IV (1524) und Ordo V (vor dem 15. Jh.) finden sich die gleichlautenden Rubriken «si mortuus esset, si fuerit pauper». Schon der Begriffswandel von Lazarus über das neutrale leprosus zu pauper drückt den Rechtsverlust des Kranken aus, denn pauper bedeutet in diesem Zusammenhang eindeutig macht- und rechtlos. Vgl. hierzu K.Bosl, Potens und Pauper. Begriffsgeschichtliche Studien zur gesellschaftlichen Differenzierung im frühen Mittelalter und zum «Pauperismus» des Hochmittelalters. In: Frühformen der Gesellschaft im mittelalterlichen Europa, München/Wien 1964, 106-134.

Acta Sanctorum, Sept.1, 166 bringt in der Verena-Vita eine Stelle, in der die Leprosen gleichlautend mit den «pauperes Christi» genannt sind. Zu den pauperes Christi im Gegensatz zu den pauperes cum Lazaro und den pauperes cum Petro siehe A. Lazzarino del Grosso, Armut und Reichtum im Denken Gerhohs von Reichersberg, München 1973, 24ff.

54 MGH Script. XVI, Burchardi Praepositi Urspergensis Chronika, 59.

${ }^{55} \mathrm{Zu}$ Marsilius von Padua (ca. 1275/80-1342/43) siehe das Nachwort in der gekürzten, von H. Rausch herausgegebenen deutschen Ausgabe des Defensor pacis, Der Verteidiger des Friedens, Stuttgart 1971, 214-241, mit zahlreichen Literaturangaben. 
${ }^{56}$ Vgl. R. Scholz, Marsilius von Padua und die Genesis des modernen Staatsbewußtseins. In: HZ 156 (1936), 88-103.

${ }^{57}$ MGH Leg. IV, Const. VIII, 252 f., $\S \S 9 / 10$; ferner 568, § 24.

${ }^{58}$ Es ist anzunehmen, daß Marsilius die Ergebnisse der Lepraforschung seiner Zeit bekannt gewesen sind und sein Urteil maßgeblich beeinflußt haben. Zur Bedeutung der Mediziner Bernard de Gordon, vor allem aber des Chirurgen Guy de Chauliac $(\dagger 1368)$ für die Lepradiagnose vgl. A. Stettler-Schär, Leprologie, $66 \mathrm{ff}$.

${ }^{59}$ J.Sydow, Kanonistische Fragen zur Geschichte des Spitals in Südwestdeutschland. In: HJb 83 (1964), 54-68.

${ }^{60}$ A. Mitterwieser, Verzeichnis, a.a. O. (Anm. 49).

${ }^{61}$ F. Merzbacher, Leprosen, a. a. O., 43 ff. (Anm. 8).

${ }^{62}$ F. Meffert, Caritas, a. a. O., $121 \mathrm{f}$. (Anm.10).

${ }^{63}$ F.X.Bärlehner, Die Entwicklung der karitativen Wohlfahrtspflege in Bayern, Nürnberg $1927,138$.

${ }^{64}$ Mündliche Mitteilung von Herrn Univ. Prof. DDr. W. Dürig in München.

\section{Summary}

The history of leprosy in Europe from the early Middle Ages to the beginning of the 16th century is at the same time the history of a religious taboo. The European society of the 10th and 11th century was compelled to create a new social state for the leper, like that of monks or other sacred persons; by this means it was possible to restrict leprosy but also to take care of the lepers. The critical and enlightened mentality, propagated vehemently in the 15th century, consequently secularized the taboo, sheltering leprosy and well endowed institutions for lepers the centuries before.

Dr. Hermann Hörger

Deutschherrnplatz 3

D-8897 Pöttmes-Echsheim 